Medtronic. Brad Bohnstedt, Richard Bellon, Harris Hawk: Nothing to disclose.

\section{EP21 HOW FAR CAN WE GO? WEB TECHNOLOGY FOR THE TREATMENT OF SIDEWALL INTRACRANIAL ANEURYSMS: INITIAL SINGLE CENTER EXPERIENCE}

B Pabon, V Torres, M Patiño, O Vargas, J Pelaez, N Serna, J Mejia. Angioteam, Medellin, Colombia

10.1136/neurintsurg-2021-ESMINT.21

Materials and Methods Patients were carefully selected. IRB approved. Clinical, anatomical, angiographical, and technical considerations were analyzed. Procedure-related complications, procedural time, antiplatelet therapy requirements. Web Occlusion Scale (WOS) was used for the Follow-up.

Results From August 2017 and March 2021 a total of 14 wide-necked, sidewall, IA were selected for WEB treatment. Aneurysm mean size $5.3 \mathrm{~mm}$ in width and 5.8 in height.

Aneurysm Location: ICA 8 cases (five PComA, two Carotid-ophthalmic segment, one AChoA segment), Superior Cerebellar Artery SCA in 5 patients (35\%), and one impressive case in posterior circulation associated with a basilar fenestration next to VBJ. Eight cases were unruptured (57\%), and six cases with a history of SAH-acute setting. DAPT was used preoperatively in all elective cases but no patient remained under antiplatelets after the procedure. Technical success of $100 \%$. Mean procedure time: $24 \mathrm{~min}$. None related procedure complications were recorded. Immediately angiographic occlusion was evidenced in 9 cases. Radiological Follow up (ranging 1 26 months) available in 9/14 showed a WOS adequate occlusion in all cases.

Conclusion In our early experience using WEB device to treat different conditions than bifurcation intracranial aneurysms, the results showed that the endossacular approach was feasible in highly selected patients, the safety profile in agreement with previous bifurcation experiences, and very effective to treat challenge cases with a high probability of recurrence or therapeutic failure.

\section{REFERENCES}

1. Arthur AS, et al. The safety and effectiveness of the Woven EndoBridge (WEB) system for the treatment of wide-necked bifurcation aneurysms: final 12-month results of the pivotal WEB intrasaccular therapy (WEB-IT) study. I Neurointerv Surg 2019. PMID: 30992395.

2. Goyal N, et al. Free PMC article. Clinical trial. How to WEB: a practical review of methodology for the use of the Woven EndoBridge. I Neurointerv Surg 2020. PMID: 32005760 Free PMC article. Review.

Disclosure Boris Pabon proctorship con MEDTRONIC, Microvention Consultant MIVI

\section{EP22 THE WALLABY AVENIR COIL SYSTEM: INITIAL EXPERIENCE WITH USE OF AVENIR COILS FOR TREATMENT OF INTRACRANIAL ANEURYSMS}

J Yeomans, A Sastry. Department of Neuroradiology, University Hospital of Wales, Cardiff, UK

10.1136/neurintsurg-2021-ESMINT.22

Introduction The Wallaby Avenir coil system is a novel generation of platinum embolic coil system indicated for endovascular embolization of intracranial aneurysms and other neurovascular abnormalities. The coils come in framing, filling and finishing types in different sizes both in helical and three dimensional shapes. The unique feature of this coil is that it does not need a detacher and can be mechanically detached with fingers once optimal coil position is achieved. This is a new move in technology that has not been recommended for reliable detachment in other pre-existing coil embolization systems.

Aims To assess Wallaby Avenir coil embolization system detachment reliability, summarise intra-procedural outcomes in the treatment of intracranial aneurysms following its introduction in a tertiary centre.

Methods This prospective study reviewed total of 11 patients with 10 intracranial aneurysms and 1 carotico-cavernous fistula between January 2021 and May 2021. 7 acute and 4 elective aneurysms were treated. All patients with intracranial aneurysms treated with Avenir coil system were included in the study.

Results The average age of the cohort was 59.5 years. $85.8 \%$ (67/78) coils Avenir coils detached reliably. Good RaymondRoy occlusion was achieved in 91\% (10/11) aneurysms in the immediate post procedural run. There was no mortality or neurological deterioration in the series. There was no intraprocedural aneurysmal rupture from Avenir coils.

Conclusions The Wallaby Avenir coil system have excellent intraprocedural safety prolife with no procedure related mortality or morbidity and good postprocedural aneurysm occlusion rates.

Disclosure Nothing to disclose

\section{Brain AVM/AVF, spinal vascular malformations}

\section{EP23* ENDOVASCULAR TREATMENT OF ANTERIOR CRANIAL FOSSA FISTULAS: THE SIGNIFICANCE OF RETROGRADE TRANSVENOUS APPROACH}

B Pabon, M Patiño, C Estrada, V Torres, N Serna, J Pelaez, 0 Vargas, J Mejia. Angioteam Medellin, Colombia

\subsection{6/neurintsurg-2021-ESMINT.23}

Materials and Methods Between September 2016 and March 2019 a retrospective review was performed. A total of 9 Patients with DAVF of the anterior cranial fossa managed with embolization through the venous side with Onyx/PHIL were selected.

Results Nine patients were included in this study, patients were between 14 and 79 years old (mean 45.6). Six primarily presented with intracranial hemorrhage. All fistulas were fed by the bilateral ethmoidal arteries arising from the ophthalmic artery and by the anterior branch of the middle meningeal artery. One case with history of type D CCF. The abnormal shunt drained into the superior sagittal sinus with the interposition of the cortical veins in all nine patients. All of the cases had high-grade Cognard classifications (III-IV). 4 (44\%) patients had been treated via transarterial embolization (TAE) via the AEA of the OA. All cases were treated via transvenous embolization (TVE), 8 of 9 (88\%) were treated with the trans-SSS approach. A complete angiographic cure was achieved in all patients, without postprocedural complications. There were nearly no symptoms among the patients during follow-up. 